\title{
Estimates of the rate of convergence for certain quadrature formulas on the half-line
}

\author{
Adhemar Bultheel, Carlos Díaz-Mendoza, Pablo González-Vera, \\ and Ramon Orive
}

Dedicated to Jerry Lange on the occasion of his 70th birthday

\begin{abstract}
We investigate the rate of convergence of so-called $n$-point Gauss type quadrature formulas to integrals of the form $\int_{0}^{\infty} f(x) \mathrm{d} \alpha(x)$ where $\alpha$ is a general distribution function on $[0, \infty)$ and where $f$ is analytic and admits a Laurent expansion in $\mathbb{C} \backslash\{0\}$. The general results are then applied in the special case where $\operatorname{d} \alpha(x)=x^{a} \exp \left\{-\left(x^{\gamma}+x^{-\gamma}\right)\right\} \mathrm{d} x, a \in \mathbb{R}$ and $\gamma \in(1 / 2, \infty)$.
\end{abstract}

\section{Introduction}

Let $\alpha$ be a general distribution function on $[0, \infty)$. We consider quadrature formulas of the form $\sum_{j=1}^{n} \lambda_{j n} f\left(x_{j n}\right)$ with positive weights $\lambda_{j n}>0$ and with real knots $x_{j n}>0$. If these quadrature formulas are exact for all integrals $I_{\alpha}(f)=$ $\int_{0}^{\infty} f(x) \mathrm{d} \alpha(x)$ where $f$ is an arbitrary Laurent polynomial of the form $\sum_{j=-p}^{q} a_{j} x^{j}$ where $p=p(n)$ and $q=q(n)$ are nonnegative integers such that $p+q=2 n-1$, then these are called $n$-point Gauss type quadrature formulas. If we want to investigate the behavior of these quadrature formulas as $n \rightarrow \infty$ with both $p(n)$ and $q(n)$ tending to $\infty$, then it is natural to consider integrals $\int_{0}^{\infty} f(x) \mathrm{d} \alpha(x)$ where $f(z)=$ $\sum_{-\infty}^{\infty} f_{k} z^{k}$ such that $\sum_{k=0}^{\infty} f_{k} z^{k}$ and $\sum_{k=1}^{\infty} f_{-k} z^{k}$ represent entire functions. This class generalizes in a natural way the Laurent polynomials.

In this paper we shall give rates of convergence for these $n$-point Gauss-type quadrature formulas. In Section 2 we introduce the necessary notation and definitions. The main results are introduced in Section 3 and in Section 4 we apply these general results to the special case when $\mathrm{d} \alpha(x)=x^{a} \exp \left\{-\left(x^{\gamma}+x^{-\gamma}\right)\right\} \mathrm{d} x, a \in \mathbb{R}$ and $\gamma \in(1 / 2, \infty)$.

1991 Mathematics Subject Classification. Primary 65D30; Secondary 41A21.

The work of the first author was performed as part of the project "Orthogonal systems and their applications" of the FWO under grant \#G0278.97.

The work of the other three authors was supported by the scientific research project of the Spanish D.G.I.C.Y.T. under contract PB96-1029. 


\section{Notation and definitions}

Let $\alpha(x)$ be a distribution function on $[0, \infty)$, i.e. a real valued, nondecreasing function with infinitely many points of increase such that the moment function

$$
m(t)=\int_{0}^{\infty} x^{t} \mathrm{~d} \alpha(x), \quad t \in \mathbb{R}
$$

exists and is finite in the Lebesgue-Stieltjes sense. Define the moments

$$
c_{k}=m(k)=\int_{0}^{\infty} x^{k} \mathrm{~d} \alpha(x), \quad k \in \mathbb{Z} .
$$

For simplicity, we assume that $c_{0}=1$. Denote

$$
I_{\alpha}(f)=\int_{0}^{\infty} f(x) \mathrm{d} \alpha(x)
$$

whenever the Lebesgue-Stieltjes integral is defined and finite. Let $\{p(n)\}_{n \in \mathbb{N}}$ be a sequence of nonnegative integers such that $0 \leq p(n) \leq 2 n-1$ for any $n$. Now, because of (2.1), all the moments $c_{k}, k \in \mathbb{Z}$ are finite and a polynomial $Q_{n}$ of exact degree $n$ (unique up to a multiplicative factor) can be constructed satisfying

$$
\int_{0}^{\infty} x^{j} Q_{n}(x) x^{-p(n)} \mathrm{d} \alpha(x)=0, \quad j=0,1, \ldots, n-1 .
$$

Let $\left\{x_{j n}\right\}_{j=1}^{n}$ be the zeros of $Q_{n}$, then there exist positive weights $\left\{A_{j n}\right\}_{j=1}^{n}$ such that

$$
\int_{0}^{\infty} P(x) x^{-p(n)} \mathrm{d} \alpha(x)=\sum_{j=1}^{n} A_{j n} P\left(x_{j n}\right), \quad \forall P \in \Pi_{2 n-1}
$$

where $\Pi_{k}(k \geq 0)$ denotes the space of polynomials of degree $k$ at most. For $p$ and $q$ nonnegative integers, we write for subspaces of Laurent polynomials

$$
\Lambda_{-p, q}=\left\{L(x)=\sum_{j=-p}^{q} a_{j} x^{j}\right\} \subset \Lambda
$$

where $\Lambda$ is the space of all Laurent polynomials. Take $L \in \Lambda_{-p(n), q(n)}(p(n)+q(n)=$ $2 n-1)$, then, since $L(x)=x^{-p(n)} P(x)$ with $P \in \Pi_{2 n-1}$, one has

$$
\int_{0}^{\infty} L(x) \mathrm{d} \alpha(x)=\int_{0}^{\infty} P(x) x^{-p(n)} \mathrm{d} \alpha(x)=\sum_{j=1}^{n} A_{j n} P\left(x_{j n}\right)=\sum_{j=1}^{n} \lambda_{j n} L\left(x_{j n}\right),
$$

where $\lambda_{j n}=x_{j n}^{p(n)} A_{j n}>0, j=1, \ldots, n$. The expression

$$
I_{n}(f)=\sum_{j=1}^{n} \lambda_{j n} f\left(x_{j n}\right)
$$

will be called the $n$-point Gauss-type quadrature formula for $\mathrm{d} \alpha(x)$ in $\Lambda_{-p(n), q(n)}$. Observe that $I_{\alpha}(L)=I_{n}(L)$ for any $L \in \Lambda_{-p(n), q(n)}$. For further details about these quadrature formulas see [BDMGVO97a],[BDMGVO97b],[BDMGVO98].

In this paper we shall be concerned with the convergence of these quadrature formulas for functions admitting a Laurent expansion valid in $\mathbb{C} \backslash\{0\}$. That is we 
study the convergence of $I_{n}(f)$ when $f$ is a Lebesgue-Stieltjes integrable function with respect to $\mathrm{d} \alpha(x)$ on $[0, \infty)$ which is of the form

$$
f(z)=f^{+}(z)+f^{-}(z), \text { with } f^{+}(z)=\sum_{j=0}^{\infty} f_{j}^{+} z^{j} \text { and } f^{-}(z)=\sum_{j=1}^{\infty} f_{j}^{-} z^{-j},
$$

$f^{+}(z)$ and $f^{-}(1 / z)$ being entire functions. This class of functions $f$ is clearly the most natural generalization of the Laurent polynomials.

We now introduce two functions $R_{x}$ and $r_{x}$ which we shall use to control the size of respectively the largest and smallest node $x_{j n}$ as $n \rightarrow \infty$. These $R_{x}$ and $r_{x}$ are two arbitrary functions defined on $[1, \infty)$ which satisfy the following conditions: $R_{x}>0$ and $r_{x}>0, R_{x}$ is monotonically increasing and $r_{x}$ is monotonically decreasing, and

$$
\begin{gathered}
\lim _{x \rightarrow \infty} R_{x}=\infty \text { and } \max \left\{x_{j n}: 1 \leq j \leq n\right\} \leq R_{n}, \\
\lim _{x \rightarrow \infty} r_{x}=0 \text { and } \min \left\{x_{j n}: 1 \leq j \leq n\right\} \geq r_{n} .
\end{gathered}
$$

Thus $x_{j n} \in\left[r_{n}, R_{n}\right], j=1, \ldots, n, n \in \mathbb{N}$.

In the rest of this paper we also assume that the sequences $p(n)$ and $q(n)$ are both monotonically increasing and that they satisfy

$$
\lim _{n \rightarrow \infty} p(n)=\lim _{n \rightarrow \infty} q(n)=\infty \text {. }
$$

Next, we introduce two monotonically increasing functions which will measure the degree of exactness of the formulas $I_{n}(f)$. Set

$$
v(x)=\min \{n: q(n) \geq x\}, \quad x \geq q(1)
$$

and

$$
v^{*}(x)=\min \{n: p(n) \geq x\}, \quad x \geq p(1) .
$$

This gives the degree of exactness since

$$
I_{\alpha}\left(x^{j}\right)=I_{v(j)}\left(x^{j}\right) \quad \text { and } \quad I_{\alpha}\left(x^{-j}\right)=I_{v^{*}(j)}\left(x^{-j}\right), \quad \forall j \in \mathbb{N} .
$$

Assume that

$$
\lim _{n \rightarrow \infty} \frac{p(n)}{2 n}=\theta \in(0,1) .
$$

By (2.7) it immediately follows that

$$
q(v(n)-1)<n \leq q(v(n)) .
$$

Hence

$$
\frac{q(v(n)-1)}{2 v(n)}<\frac{n}{2 v(n)} \leq \frac{q(v(n))}{2 v(n)} .
$$

Since $\lim _{n \rightarrow \infty} v(n)=\infty$ (recall that $\left.\lim _{n \rightarrow \infty} q(n)=\infty\right)$, we get

$$
\lim _{n \rightarrow \infty} \frac{n}{2 v(n)}=1-\theta \text {. }
$$

Similarly, by (2.8) and (2.9), one obtains

$$
\lim _{n \rightarrow \infty} \frac{n}{2 v^{*}(n)}=\theta
$$


The functions which we introduce next, will enable us to formulate convergence results to be given in Section 3 in a more elegant form. These functions are

$$
\psi(\theta)=\varlimsup_{n \rightarrow \infty} \frac{R_{v(n)}}{R_{\frac{n}{2(1-\theta)}}}
$$

and

$$
\psi^{*}(\theta)=\varliminf_{n \rightarrow \infty} \frac{r_{v^{*}(n)}}{r_{\frac{n}{2 \theta}}} .
$$

Furthermore we introduce

$$
\mu(\theta)=\varlimsup_{n \rightarrow \infty} \frac{\gamma_{n}^{1 / q(n)}}{R_{\frac{q(n)}{2(1-\theta)}}}
$$

where

$$
\gamma_{n}=\int_{0}^{\infty} Q_{n}(x)^{2} x^{-p(n)} \mathrm{d} \alpha(x)
$$

and $Q_{n}$ the $n$th monic orthogonal polynomial for $x^{-p(n)} \mathrm{d} \alpha(x)$. We also need the so called Christoffel function of degree $m$ for the distribution $\mathrm{d} \alpha_{n}(x)=x^{-(p(n)+1)} \mathrm{d} \alpha(x)$ as given by

$$
K_{m}\left(z, \alpha_{n}\right)=\sup _{P \in \Pi_{m}} \frac{|P(z)|^{2}}{\|P\|_{2}^{2}}
$$

where $\|P\|_{2}^{2}=\int_{0}^{\infty}|P(x)|^{2} \mathrm{~d} \alpha_{n}(x)$. This allows us to define

$$
\kappa(\theta)=\varlimsup_{n \rightarrow \infty} \frac{r_{\frac{p(n)}{2 \theta}}}{\left[K_{n}\left(0, \alpha_{n}\right)\right]^{1 / p(n)}} .
$$

For any entire function $f(z)=\sum_{j=0}^{\infty} f_{j} z^{j}$, we define its order by

$$
\rho(f)=\varlimsup_{n \rightarrow \infty} \frac{\log n^{-n}}{\log \left|f_{n}\right|}
$$

and if $0<\rho(f)<\infty$, its type is

$$
\tau(f)=\varlimsup_{n \rightarrow \infty} \frac{n\left|f_{n}\right|^{\rho(f) / n}}{\mathrm{e} \rho(f)}, \quad \mathrm{e}=\exp (1) .
$$

Finally, for $s>0$, the indices $\sigma_{R}$ and $\sigma_{r}$ are defined by

$$
\begin{aligned}
\sigma_{R}(f ; s) & =\varlimsup_{n \rightarrow \infty}\left|f_{n}\right|^{1 / n} R_{s n} \\
\sigma_{r}(f ; s) & =\varlimsup_{n \rightarrow \infty}\left|f_{n}\right|^{1 / n} / r_{s n} .
\end{aligned}
$$

For more information and properties on several of the notions that were introduced, we refer to [Mha96]. There one can also find in greater detail several (polynomial) results that are related to some of the problems we discuss in this paper for the case of Laurent polynomials. 


\section{Rates of convergence}

In this section we give the main results about the convergence of the quadrature formulas $I_{n}(f), n=1,2, \ldots$ as defined in Section 2. To understand better the technical aspects, we start with two lemmas which give the relation between the parameters $\rho, \tau$ and $\sigma$. They are basically contained in [Lub83].

LEMma 3.1. Let $f^{+}(z)=\sum_{j=0}^{\infty} f_{j}^{+} z^{j}$ be an entire function.

a) Suppose that for some $q^{+}>0$ and $c^{+}>0, R_{x} \leq\left(c^{+} x\right)^{q^{+}}$for sufficiently large $x$. Then

i) $\rho\left(f^{+}\right)<1 / q^{+} \Rightarrow \sigma_{R}\left(f^{+} ; s\right)=0, \forall s>0$

ii) $\rho\left(f^{+}\right)=1 / q^{+} \Rightarrow \sigma_{R}\left(f^{+} ; s\right) \leq\left(c^{+} s \text { e } \tau\left(f^{+}\right) / q^{+}\right)^{q^{+}}, \forall s>0$

b) Suppose that for some $q_{1}^{+}>0$ and $c_{1}^{+}>0, R_{x} \geq\left(c_{1}^{+} x\right)^{q_{1}^{+}}$for sufficiently large $x$. Then

i) $\rho\left(f^{+}\right)>1 / q_{1}^{+} \Rightarrow \sigma_{R}\left(f^{+} ; s\right)=\infty, \forall s>0$

ii) $\rho\left(f^{+}\right)=1 / q_{1}^{+} \Rightarrow \sigma_{R}\left(f^{+} ; s\right) \geq\left(c_{1}^{+} s \text { e } \tau\left(f^{+}\right) / q_{1}^{+}\right)^{q_{1}^{+}}, \forall s>0$

REMARK 3.1. If $R_{x}=(K x)^{q^{+}}$, then equality holds in a)ii) and in b)ii), so that $\sigma_{R}\left(f^{+} ; s\right)$ can be estimated exactly in terms of $\tau\left(f^{+}\right)$.

The following lemma gives the analog for entire functions in $1 / z$.

Lemma 3.2. Let $f^{-}(z)=\sum_{j=1}^{n} f_{j}^{-} z^{-j}$ be an entire function.

a) Suppose that for some $q^{-}>0$ and $c^{-}>0,1 / r_{x} \leq\left(c^{-} x\right)^{q^{-}}$for sufficiently large $x$. Then

i) $\rho\left(f^{-}\right)<1 / q^{-} \Rightarrow \sigma_{r}\left(f^{-} ; s\right)=0, \forall s>0$

ii) $\rho\left(f^{-}\right)=1 / q^{-} \Rightarrow \sigma_{r}\left(f^{-} ; s\right) \leq\left(c^{-} s \text { e } \tau\left(f^{-}\right) / q^{-}\right)^{q^{-}}, \forall s>0$

b) Suppose that for some $q_{1}^{-}>0$ and $c_{1}^{-}>0,1 / r_{x} \geq\left(c_{1}^{-} x\right)^{q_{1}^{-}}$for sufficiently large $x$. Then

i) $\rho\left(f^{-}\right)>1 / q_{1}^{-} \Rightarrow \sigma_{r}\left(f^{-} ; s\right)=\infty, \forall s>0$

ii) $\rho\left(f^{-}\right)=1 / q_{1}^{-} \Rightarrow \sigma_{r}\left(f^{-} ; s\right) \geq\left(c_{1}^{-} s \text { e } \tau\left(f^{-}\right) / q_{1}^{-}\right)^{q_{1}^{-}}, \forall s>0$

Now, taking into account that $q(n) \geq 0$, we have $\sum_{j=1}^{n} \lambda_{j n}=c_{0}=1$. Thus, from the definition of $R_{x}$ and $r_{x}$, the following lemma can de deduced.

LEMMA 3.3. Let $I_{n}(f)=\sum_{j=1}^{n} \lambda_{j n} f\left(x_{j n}\right)$ denote the $n$-point Gauss-type quadrature formula for $I_{\alpha}(f)$ in $\Lambda_{-p(n), q(n)},(p(n)+q(n)=2 n-1)$. Then

a) $I_{n}\left(x^{j}\right) \leq\left[R_{n}^{\frac{\operatorname{sign}(j)+1}{2}} / r_{n}^{\frac{1-\operatorname{sign}(j)}{2}}\right]^{|j|}, n \in \mathbb{N}, j \in \mathbb{Z}$, where

$$
\operatorname{sign}(x)=\left\{\begin{aligned}
1, & \text { for } x \geq 0 \\
-1, & \text { for } x<0
\end{aligned}\right.
$$

b) $I_{\alpha}\left(x^{j}\right)=I_{v(j)}\left(x^{j}\right) \leq R_{v(j)}^{j}, \quad j \in \mathbb{N}$.

c) $I_{\alpha}\left(x^{-j}\right)=I_{v^{*}(j)}\left(x^{-j}\right) \leq r_{v^{*}(j)}^{-j}, \quad j \in \mathbb{N}$.

We prove one more lemma. 
Lemma 3.4. Assume that $f=f^{+}+f^{-}$is given as in (2.4). Furthermore suppose that $\psi(\theta)$ and $1 / \psi^{*}(\theta)$ as given by (2.12) and (2.13) are both finite. Assume also that $\sigma_{R}\left(f_{1}^{+} ; \frac{1}{2(1-\theta)}\right) \psi(\theta)<1$ and $\sigma_{r}\left(f^{-} ; \frac{1}{2 \theta}\right) / \psi^{*}(\theta)<1$. Then

$$
I_{\alpha}(f)=\sum_{j=0}^{\infty} f_{j}^{+} I_{\alpha}\left(z^{j}\right)+\sum_{j=1}^{\infty} f_{j}^{-} I_{\alpha}\left(z^{-j}\right)
$$

Proof. We have to prove that

$$
I_{\alpha}\left(\sum_{j=0}^{\infty} f_{j}^{+} z^{j}\right)=\sum_{j=0}^{\infty} f_{j}^{+} I_{\alpha}\left(z^{j}\right) \quad \text { and } \quad I_{\alpha}\left(\sum_{j=1}^{\infty} f_{j}^{-} z^{-j}\right)=\sum_{j=1}^{\infty} f_{j}^{-} I_{\alpha}\left(z^{-j}\right) .
$$

We shall only prove the second equality since the first one is proved in a completely similar way. By the dominated convergence theorem [Rud74, Theorem 1.34], it is sufficient to prove that $\sum_{j=1}^{\infty}\left|f_{j}^{-} x^{-j}\right|$ is a Lebesgue-Stieltjes integrable function with respect to $\mathrm{d} \alpha(x)$ on $[0, \infty)$. Since $f^{-}(z)=\sum_{j=1}^{\infty} f_{j}^{-} z^{-j}$ is holomorphic in $\hat{\mathbb{C}} \backslash\{0\}$ where $\hat{\mathbb{C}}=\mathbb{C} \cup\{\infty\}$ is the extended complex plane, we can write

$$
\int_{0}^{\infty} \sum_{j=1}^{\infty}\left|f_{j}^{-}\right| x^{-j} \mathrm{~d} \alpha(x)=\lim _{b \downarrow 0} \int_{b}^{\infty} \sum_{j=1}^{\infty}\left|f_{j}^{-}\right| x^{-j} \mathrm{~d} \alpha(x)=\lim _{b \downarrow 0} \sum_{j=1}^{\infty}\left|f_{j}^{-}\right| \int_{b}^{\infty} x^{-j} \mathrm{~d} \alpha(x) .
$$

Because $\int_{b}^{\infty} x^{-j} \mathrm{~d} \alpha(x) \leq \int_{0}^{\infty} x^{-j} \mathrm{~d} \alpha(x), b>0$, it results that

$$
\int_{0}^{\infty} \sum_{j=1}^{\infty}\left|f_{j}^{-}\right| x^{-j} \mathrm{~d} \alpha(x) \leq \sum_{j=1}^{\infty}\left|f_{j}^{-}\right| I_{\alpha}\left(x^{-j}\right) .
$$

Thus we have to check that the series $\sum_{j=1}^{\infty} f_{j}^{-} I_{\alpha}\left(x^{-j}\right)$ is absolutely convergent. Now by c) in Lemma 3.3, one has

$$
\left|f_{j}^{-}\right|\left|I_{\alpha}\left(x^{-j}\right)\right|=\left|f_{j}^{-}\right|\left|I_{v^{*}(j)}\left(x^{-j}\right)\right| \leq\left|f_{j}^{-}\right| r_{v^{*}(j)}^{-j}
$$

which gives

$$
\left|f_{j}^{-}\right|^{1 / j}\left|I_{\alpha}\left(x^{-j}\right)\right|^{1 / j} \leq\left|f_{j}^{-}\right|^{1 / j} r_{v^{*}(j)}^{-1} .
$$

Therefore

$$
\begin{aligned}
\varlimsup_{j \rightarrow \infty}\left|f_{j}^{-}\right|^{1 / j}\left|I_{\alpha}\left(x^{-j}\right)\right|^{1 / j} & \leq \varlimsup_{j \rightarrow \infty}\left|f_{j}^{-}\right|^{1 / j}\left[r_{\frac{j}{2 \theta}}^{-1}\right] \frac{\left[r_{v^{*}(j)}^{-1}\right]}{\left[r_{\frac{j}{2 \theta}}^{-1}\right]} \\
& \leq \sigma_{r}\left(f^{-} ; \frac{1}{2 \theta}\right) / \psi^{*}(\theta) .
\end{aligned}
$$

To simplify the notation we set $s=s(\theta)=\sigma_{r}\left(f^{-} ; \frac{1}{2 \theta}\right) / \psi^{*}(\theta)$. (Recall that by assumption $s<1$.) Thus, there exists some $n_{0}$ such that for all $n \geq n_{0}$

$$
\sup _{j \geq n}\left|f_{j}^{-}\right|^{1 / j}\left|I_{\alpha}\left(x^{-j}\right)\right|^{1 / j} \leq s+\epsilon<1, \quad \epsilon>0 .
$$

Choose $p>0$ such that $\eta=\eta(\theta)=p s=s+\epsilon<1$. Then

$$
\sum_{j=n+1}^{\infty}\left|f_{j}^{-}\right|^{1 / j}\left|I_{\alpha}\left(x^{-j}\right)\right|^{1 / j} \leq \eta^{n+1} \sum_{k=0}^{\infty} \eta^{k}=\frac{\eta^{n+1}}{1-\eta}
$$

Thus the series $\sum_{j=1}^{\infty} f_{j}^{-} I_{\alpha}\left(x^{-j}\right)$ converges absolutely and the lemma is proved. 
We are now in a position to state the first result concerning the estimate of the rate of convergence of the sequence $I_{n}(f), n=1,2, \ldots$ of Gauss-type quadrature formulas.

THEOREM 3.5. Let $f(z)$ be a function satisfying (2.4) and suppose that $\psi(\theta)$ and $1 / \psi^{*}(\theta)$ as given by (2.12) and (2.13) are both finite. Assume also that $\sigma_{R}\left(f_{1}^{+} ; \frac{1}{2(1-\theta)}\right) \psi(\theta)<1$ and $\sigma_{r}\left(f^{-} ; \frac{1}{2 \theta}\right) / \psi^{*}(\theta)<1$. Then

$$
\varlimsup_{n \rightarrow \infty}\left|I_{\alpha}(f)-I_{n}(f)\right|^{1 / 2 n} \leq \max \left\{\eta^{+}, \eta^{-}\right\}
$$

where

$$
\eta^{+}=\left[\sigma_{R}\left(f^{+} ; \frac{1}{2(1-\theta)}\right) \psi(\theta)\right]^{1-\theta} \quad \text { and } \quad \eta^{-}=\left[\sigma_{r}\left(f^{-} ; \frac{1}{2 \theta}\right) / \psi^{*}(\theta)\right]^{\theta} .
$$

Proof. By (2.4), set $f=f^{+}+f^{-}$with $f^{+}(z)=\sum_{j=0}^{\infty} f_{j}^{+} z^{j}$ and $f^{-}(z)=$ $\sum_{j=1}^{\infty} f_{j}^{-} z^{-j}$. Thus, with $E_{n}=I_{\alpha}-I_{n}$,

$$
\begin{aligned}
E_{n}(f) & =E_{n}\left(f^{+}\right)-E_{n}\left(f^{-}\right) \\
& =I_{\alpha}\left(f^{+}\right)-\sum_{j=0}^{\infty} f_{j} I_{n}\left(x^{j}\right)+I_{\alpha}\left(f^{-}\right)-\sum_{j=1}^{\infty} f_{j}^{-} I_{n}\left(x^{-j}\right) .
\end{aligned}
$$

By the previous lemma, we know that

$$
I_{\alpha}\left(\sum_{j=0}^{\infty} f_{j}^{+} x^{j}\right)=\sum_{j=0}^{\infty} f_{j}^{+} I_{\alpha}\left(x^{j}\right) \quad \text { and } \quad I_{\alpha}\left(\sum_{j=1}^{\infty} f_{j}^{-} x^{-j}\right)=\sum_{j=1}^{\infty} f_{j}^{-} I_{\alpha}\left(x^{-j}\right) .
$$

Thus, since $I_{n}(f)=I_{\alpha}(f), \forall f \in \Lambda_{-p(n), q(n)}$, we can write

$$
E_{n}(f)=\sum_{j=q(n)+1}^{\infty} f_{j}^{+} E_{n}\left(x^{j}\right)+\sum_{j=p(n)+1}^{\infty} f_{j}^{-} E_{n}\left(x^{-j}\right) .
$$

We study the convergence of both series in the right-hand side separately. First we consider the series $\sum_{j=q(n)+1}^{\infty} f_{j}^{+} E_{n}\left(x^{j}\right)$. From the definition of $v(x)$ in (2.7), Lemma 3.3, and the monotonicity of $R_{x}$, it follows that

$$
\begin{aligned}
\sup _{j>q(n)}\left|f_{j}^{+}\right|^{1 / j}\left|I_{\alpha}\left(x^{j}\right)-I_{n}\left(x^{j}\right)\right|^{1 / j} & =\sup _{j>q(n)}\left|f_{j}^{+}\right|^{1 / j}\left|I_{v(j)}\left(x^{j}\right)-I_{n}\left(x^{j}\right)\right|^{1 / j} \\
& \leq \sup _{j>q(n)}\left|f_{j}^{+}\right|^{1 / j}\left(R_{v(j)}^{j}+R_{n}^{j}\right)^{1 / j} \\
& \leq 2^{1 / q(n)} \sup _{j>q(n)}\left|f_{j}^{+}\right|^{1 / j} R_{v(j)} .
\end{aligned}
$$

Then

$$
\begin{aligned}
\varlimsup_{n \rightarrow \infty} \sup _{j>q(n)}\left|f_{j}^{+}\right|^{1 / j}\left|E_{n}\left(x^{j}\right)\right|^{1 / j} & \leq \lim _{n \rightarrow \infty} \sup _{j>q(n)}\left|f_{j}^{+}\right|^{1 / j} R_{v(j)} \\
& =\lim _{n \rightarrow \infty} \sup _{j>q(n)}\left|f_{j}^{+}\right|^{1 / j} R_{\frac{j}{2(1-\theta)}} \frac{R_{v(j)}}{R_{\frac{j}{2(1-\theta)}}} \\
& \leq \sigma_{R}\left(f^{+} ; \frac{1}{2(1-\theta)}\right) \psi(\theta) .
\end{aligned}
$$

Observe that since $\lim _{n \rightarrow \infty} q(n)=\infty$ and $\sup _{j>q(n)}\left|f_{j}^{+}\right|^{1 / j} R_{\frac{j}{2(1-\theta)}}$ is monotonically decreasing, it holds that

$$
\lim _{n \rightarrow \infty} \sup _{j>q(n)}\left|f_{j}^{+}\right|^{1 / j} R_{\frac{j}{2(1-\theta)}}=\lim _{n \rightarrow \infty} \sup _{j>n}\left|f_{j}^{+}\right|^{1 / j} R_{\frac{j}{2(1-\theta)}}=\sigma_{R}\left(f^{+} ; \frac{1}{2(1-\theta)}\right) .
$$


We now proceed as in the proof of the previous lemma. By hypothesis, we know that $\sigma_{R}\left(f^{+} ; \frac{1}{2(1-\theta)}\right) \psi(\theta)<1$. Take $p>1$ such that $\eta=\eta(\theta):=p \sigma_{R}\left(f^{+} ; \frac{1}{2(1-\theta)}\right) \psi(\theta)<$ 1 , then there exists an $n_{0}=n_{0}(p) \in \mathbb{N}$ so that for any $n>n_{0}$ by (3.1)

$$
\sup _{j>q(n)}\left|f_{j}^{+}\right|^{1 / j}\left|E_{n}\left(x^{j}\right)\right|^{1 / j} \leq \eta<1
$$

and consequently

$$
\left|f_{j}^{+}\right|\left|E_{n}\left(x^{j}\right)\right| \leq \eta^{j}, \quad j \geq q(n)
$$

Therefore

$$
\begin{aligned}
\sum_{j=q(n)+1}^{\infty}\left|f_{j}^{+}\right|\left|E_{n}\left(x^{j}\right)\right| & \leq \sum_{j=q(n)+1}^{\infty} \eta^{j} \\
& =\eta^{q(n)+1} \sum_{k=0}^{\infty} \eta^{k}=\frac{\eta^{q(n)+1}}{1-\eta}
\end{aligned}
$$

Thus, one sees that the series $\sum_{j=q(n)+1}^{\infty}\left|f_{j}^{+} \| I_{\alpha}\left(x^{j}\right)-I_{n}\left(x^{j}\right)\right|$ is convergent and it follows that

$$
\varlimsup_{n \rightarrow \infty}\left(\sum_{j=q(n)+1}^{\infty}\left|f_{j}^{+} \| E_{n}\left(x^{j}\right)\right|\right)^{1 / 2 n} \leq \eta^{1-\theta} .
$$

As the left-hand side in the last inequality does not depend on the parameter $p$, we can replace $p$ by 1 so that

$$
\varlimsup_{n \rightarrow \infty}\left(\sum_{j=q(n)+1}^{\infty}\left|f_{j}^{+} \| E_{n}\left(x^{j}\right)\right|\right)^{1 / 2 n} \leq\left[\sigma_{R}\left(f^{+} ; \frac{1}{2(1-\theta)}\right) \psi(\theta)\right]^{1-\theta} .
$$

On the other hand, by assuming that $\sigma_{r}\left(f^{-} ; \frac{1}{2 \theta}\right) / \psi^{*}(\theta)<1$, and proceeding as above, it can be shown that

$$
\varlimsup_{n \rightarrow \infty}\left(\sum_{j=p(n)+1}^{\infty}\left|f_{j}^{-} \| E_{n}\left(x^{-j}\right)\right|\right)^{1 / 2 n} \leq\left[\sigma_{r}\left(f^{-} ; \frac{1}{2 \theta}\right) / \psi^{*}(\theta)\right]^{\theta} .
$$

Finally, by (3.2) and (3.3) and the fact that

$$
\left|E_{n}(f)\right| \leq \sum_{j=q(n)+1}^{\infty}\left|f_{j}^{+}\right|\left|E_{n}\left(x^{j}\right)\right|+\sum_{j=p(n)+1}^{\infty}\left|f_{j}^{-}\right|\left|E_{n}\left(x^{-j}\right)\right|,
$$

the proof follows.

Next, a lower bound for the rate of convergence of the sequence $\left\{I_{n}(f)\right\}$ will be given (compare with Lemma 3.8 in [Lub83]).

Lemma 3.6. Let $f$ be a function given by (2.4) with nonnegative coefficients, i.e., $f_{j}^{+} \geq 0$ and $f_{j}^{-} \geq 0$. Then

a) $I_{\alpha}(f)<\infty$ if and only if $\sum_{j=0}^{\infty} f_{j}^{+} c_{j}+\sum_{j=1}^{\infty} f_{j}^{-} c_{-j}<\infty$

b) If $I_{\alpha}(f)<\infty$, then $E_{n}(f)=I_{\alpha}(f)-I_{n}(f) \geq \gamma_{n} f_{q(n)+1}^{+}+\beta_{n} f_{p(n)+1}^{-}$where $\gamma_{n}=\int_{0}^{\infty} Q_{n}(x)^{2} x^{-p(n)} \mathrm{d} \alpha(x)$ and $\beta_{n}=Q_{n}(0)^{-2} \int_{0}^{\infty} Q_{n}(x)^{2} x^{-(p(n)+1)} \mathrm{d} \alpha(x)$. 
Proof. a) Since the partial sums in $\sum_{j=0}^{\infty} f_{j}^{+} x^{j}$ and $\sum_{j=1}^{\infty} f_{j}^{-} x^{-j}$ increase monotonically for $x \in(0, \infty)$, the proof follows from Lebesgue's monotone convergence theorem [Rud74, Theorem 1.26].

b) By part a) in this lemma, we can write

$E_{n}(f)=\sum_{j=0}^{\infty} f_{j+p(n)+1}^{-} E_{n}\left(x^{-(j+p(n)+1)}\right)+\sum_{j=0}^{\infty} f_{j+q(n)+1}^{+} E_{n}\left(x^{j+q(n)+1}\right)$.

On the other hand, from the error expansion for $I_{n}(f)$ (see [BDMGVO98])

$$
E_{n}\left(x^{k}\right) \geq 0, \quad \forall k \in \mathbb{Z} .
$$

Hence

$$
E_{n}(f) \geq f_{p(n)+1}^{-} E_{n}\left(x^{-(p(n)+1)}\right)+f_{q(n)+1}^{+} E_{n}\left(x^{q(n)+1}\right) .
$$

Let us denote by $L_{n}(\phi ; x)$ the Hermite interpolant from $\Lambda_{-p(n), q(n)}$ for the function $\phi$, at the nodes $x_{1 n}, \ldots, x_{n n}$ of the quadrature formula. By

[BDMGVO97b], it follows that

$$
E_{n}\left(x^{k}\right)=I_{\alpha}\left(x^{k}-L_{n}\left(x^{k} ; x\right)\right) .
$$

Taking $k=q(n)+1$, then

$$
x^{q(n)+1}-L_{n}\left(x^{q(n)+1} ; x\right)=x^{-p(n)} Q_{n}(x)^{2} .
$$

Therefore,

$$
I_{\alpha}\left(x^{q(n)+1}-L_{n}\left(x^{q(n)+1} ; x\right)\right)=\int_{0}^{\infty} Q_{n}(x)^{2} x^{-p(n)} \mathrm{d} \alpha(x)=\gamma_{n} .
$$

Now, for $k=-(p(n)+1)$, we have

$$
L_{n}\left(x^{-(p(n)+1)} ; x\right)=x^{-(p(n)+1)}\left[1-k_{n} Q_{n}(x)^{2}\right]
$$

with $k_{n}=Q_{n}(0)^{-2}>0$ as it can be easily checked. Hence

$$
\frac{1}{x^{p(n)+1}}-L_{n}\left(\frac{1}{x^{p(n)+1}} ; x\right)=\left[\frac{Q_{n}(x)}{Q_{n}(0)}\right]^{2} \frac{1}{x^{p(n)+1}}
$$

and this yields

$$
I_{\alpha}\left(\frac{1}{x^{p(n)+1}}-L_{n}\left(\frac{1}{x^{p(n)+1}} ; x\right)\right)=\frac{1}{Q_{n}(0)^{2}} \int_{0}^{\infty} Q_{n}(x)^{2} \frac{\mathrm{d} \alpha(x)}{x^{p(n)+1}}=\beta_{n} .
$$

The proof now follows from (3.4), (3.5) and (3.6).

We conclude this section with a number of corollaries of the previous results.

Corollary 3.7. Let $\psi(\theta)$ and $1 / \psi^{*}(\theta)$ be finite. Under the same conditions as in Lemma 3.1 and Lemma 3.2 and Theorem 3.5, the following holds.

a) If $\rho\left(f^{+}\right)<1 / q^{+}$and $\rho\left(f^{-}\right)<1 / q^{-}$, then

$$
\varlimsup_{n \rightarrow \infty}\left|I_{\alpha}(f)-I_{n}(f)\right|^{1 / 2 n}=0 .
$$


b) If $\rho\left(f^{+}\right)=1 / q^{+}$and $\rho\left(f^{-}\right)<1 / q^{-}$, then

$$
\varlimsup_{n \rightarrow \infty}\left|I_{\alpha}(f)-I_{n}(f)\right|^{1 / 2 n} \leq \eta^{+}, \quad \eta^{+}=\left[\left(\frac{c^{+} \mathrm{e} \tau\left(f^{+}\right)}{2(1-\theta) q^{+}}\right)^{q^{+}} \psi(\theta)\right]^{1-\theta}
$$

provided that $\sigma_{R}\left(f^{+} ; \frac{1}{2(1-\theta)}\right) \psi(\theta)<1$.

c) If $\rho\left(f^{+}\right)<1 / q^{+}$and $\rho\left(f^{-}\right)=1 / q^{-}$, then

$$
\varlimsup_{n \rightarrow \infty}\left|I_{\alpha}(f)-I_{n}(f)\right|^{1 / 2 n} \leq \eta^{-}, \quad \eta^{-}=\left[\left(\frac{c^{-} \mathrm{e} \tau\left(f^{-}\right)}{2 \theta q^{-}}\right)^{q^{-}} / \psi^{*}(\theta)\right]^{\theta}
$$

provided that $\sigma_{r}\left(f^{-} ; \frac{1}{2 \theta}\right) / \psi^{*}(\theta)<1$.

d) If $\rho\left(f^{+}\right)=1 / q^{+}$and $\rho\left(f^{-}\right)=1 / q^{-}$, then

$$
\varlimsup_{n \rightarrow \infty}\left|I_{\alpha}(f)-I_{n}(f)\right|^{1 / 2 n} \leq \max \left\{\eta^{+}, \eta^{-}\right\}, \quad\left(\eta^{+}, \eta^{-} \text {as above }\right)
$$

provided that $\sigma_{R}\left(f^{+} ; \frac{1}{2(1-\theta)}\right) \psi(\theta)<1$ and $\sigma_{r}\left(f^{-} ; \frac{1}{2 \theta}\right) / \psi^{*}(\theta)<1$.

REMARK 3.2. In the above corollary we have assumed that there exist positive constants $c^{+}, c^{-}, q^{+}$and $q^{-}$such that for sufficiently large $x: R_{x} \leq\left(c^{+} x\right)^{q^{+}}$and $r_{x}^{-1} \leq\left(c^{-} x\right)^{q^{-}}$. When in both of these relations equality occurs, then it can be checked that the estimates of the rate of convergence are less than 1.

Now, making use of Lemma 3.6 and definitions (2.14) and (2.17), it follows that

COROLlaRY 3.8. Let $f$ be a function as given in (2.4) with nonnegative coefficients such that $I_{\alpha}(f)<\infty$. Then

$$
\varlimsup_{n \rightarrow \infty}\left|I_{\alpha}(f)-I_{n}(f)\right|^{1 / 2 n} \geq \max \left\{\left(\sigma_{R}\left(f^{+} ; \frac{1}{2(1-\theta)}\right) \mu(\theta)\right)^{1-\theta},\left(\sigma_{r}\left(f^{-} ; \frac{1}{2 \theta}\right) \kappa(\theta)\right)^{\theta}\right\} \text {. }
$$

Finally, we can state

Corollary 3.9. Under the same hypothesis as in Lemma 3.1, Lemma 3.2, and Corollary 3.8, we have

a) If $\rho\left(f^{+}\right)>1 / q_{1}^{+}$or $\rho\left(f^{-}\right)>1 / q_{1}^{-}$, then

$$
\varlimsup_{n \rightarrow \infty}\left|I_{\alpha}(f)-I_{n}(f)\right|^{1 / 2 n}=\infty \text {. }
$$

b) If $\rho\left(f^{+}\right)=1 / q_{1}^{+}$and $\rho\left(f^{-}\right)=1 / q_{1}^{-}$, then

$$
\begin{gathered}
\varlimsup_{n \rightarrow \infty}\left|I_{\alpha}(f)-I_{n}(f)\right|^{1 / 2 n} \geq \max \left\{\eta_{1}^{+}, \eta_{1}^{-}\right\}, \\
\eta_{1}^{+}=\left[\left(\frac{c_{1}^{+} \mathrm{e} \tau\left(f^{+}\right)}{2(1-\theta) q_{1}^{+}}\right)^{q_{1}^{+}} \mu(\theta)\right]^{1-\theta}, \quad \eta_{1}^{-}=\left[\left(\frac{c_{1}^{-} \mathrm{e} \tau\left(f^{-}\right)}{2 \theta q_{1}^{-}}\right)^{q_{1}^{-}} \kappa(\theta)\right]^{\theta} .
\end{gathered}
$$

\section{An application to a family of distributions}

The general results of Section 3 will now be applied to a family of weight functions introduced in [LLMF95] and also studied in [BHMF96]. Such weight functions were also considered in our previous papers [BDMGVO97a, BDMGVO97b, BDMGV098]. Throughout this section, the distribution will be given by

$$
\mathrm{d} \alpha(x)=\omega_{\gamma}(x) \mathrm{d} x=x^{a} \exp \left\{-\left(x^{\gamma}+x^{-\gamma}\right)\right\} \mathrm{d} x, \quad x \in(0, \infty)
$$


where the parameter $a \in \mathbb{R}$ is not relevant for our results. The important parameter is $\gamma \in(1 / 2, \infty)$. Let $\left\{\lambda_{n}\right\}$ be a sequence of nonnegative integers such that $0 \leq$ $\lambda_{n} \leq 2 n$ and satisfying (2.9). Let us denote by $h_{n m}\left(x, \omega_{\gamma}\right)$ the $m$ th orthonormal polynomial with respect to $x^{-\lambda_{n}} \omega_{\gamma}(x) \mathrm{d} x$ and by $\hat{h}_{n m}\left(x, \omega_{\gamma}\right)$ the corresponding $m$ th monic orthogonal polynomial, i.e.,

$$
\hat{h}_{n m}\left(x, \omega_{\gamma}\right)=h_{n m}\left(x, \omega_{\gamma}\right) / \kappa_{n m}\left(\omega_{\gamma}\right)
$$

where $h_{n m}\left(x, \omega_{\gamma}\right)=\kappa_{n m}\left(\omega_{\gamma}\right) x^{m}+$ lower degree terms.

For our further development, and to make the paper more self-contained, we recall several results contained in [LLMF95],[BHMF96], concerning the zeros of $h_{n m}\left(x, \omega_{\gamma}\right)$ which are needed to estimate the rate of convergence.

THEOREM 4.1. $\quad$ i) Let $\left\{x_{j n}\right\}_{j=1}^{n}$ be the zeros of $h_{n n}\left(x, \omega_{\gamma}\right)$. Then, there exist positive constants $\tilde{R}_{n}, \tilde{r}_{n}$ and $K>1$ such that $x_{j n} \in\left[\tilde{r}_{n} / K, K \tilde{R}_{n}\right]$ where

$\tilde{R}_{n}=\left[\frac{2 n(1-\theta)}{\gamma B(\gamma)}\right]^{1 / \gamma}+o\left(n^{1 / \gamma}\right) \quad$ and $\quad \frac{1}{\tilde{r}_{n}}=\left[\frac{2 n \theta}{\gamma B(\gamma)}\right]^{1 / \gamma}+o\left(n^{1 / \gamma}\right)$

with $B(\gamma)=\frac{1}{\sqrt{\pi}} \frac{\Gamma(\gamma+1 / 2)}{\Gamma(\gamma+1)}$, and $\Gamma(z)$ being the Euler Gamma function.

ii) If $0 \leq \theta<1$, then

$$
\lim _{n \rightarrow \infty}\left[\kappa_{n n}\left(\omega_{\gamma}\right) n^{\frac{2 n-\lambda_{n}}{2 \gamma}}\right]^{1 / n}=\left[4\left(\frac{\mathrm{e} \gamma B(\gamma)}{2(1-\theta)}\right)^{1 / \gamma}\right]^{1-\theta} .
$$

Furthermore, if $\frac{\lambda_{n}}{2 n}=\theta+o\left(\frac{1}{\log n}\right)$ as $n \rightarrow \infty$, then

$$
\lim _{n \rightarrow \infty}\left[\kappa_{n n}\left(\omega_{\gamma}\right)\right]^{1 / n} n^{\frac{1-\theta}{\gamma}}=\left\{\begin{array}{cl}
\left(4\left[\frac{\mathrm{e} \gamma B(\gamma)}{2(1-\theta)}\right]^{1 / \gamma}\right)^{1-\theta} ; & 0 \leq \theta<1 \\
1 ; & \theta=1
\end{array}\right.
$$

Set $\Delta_{n}=\left[\tilde{r}_{n}, \tilde{R}_{n}\right]$ and (compare with $(2.16)$

$$
K_{n}\left(z, x^{-\lambda_{n}} \omega_{\gamma}\right)=\sup _{P \in \Pi_{n}} \frac{|P(z)|^{2}}{\|P\|_{2}^{2}}
$$

where $\|P\|_{2}^{2}=\int_{0}^{\infty}\left|P_{n}(x)\right|^{2} x^{-\lambda_{n}} \omega_{\gamma}(x) \mathrm{d} x$.

Let $\Phi_{\Delta_{n}}(z)$ be the conformal transformation mapping the exterior of the unit circle onto $\hat{\mathbb{C}} \backslash \Delta_{n}$, preserving the point at infinity:

$$
\Phi_{\Delta_{n}}(z)=\frac{2}{\tilde{R}_{n}-\tilde{r}_{n}}\left[z-\frac{\tilde{R}_{n}+\tilde{r}_{n}}{2}+\sqrt{T_{n}(z)}\right]
$$

with $T_{n}(z)=\left(z-\tilde{R}_{n}\right)\left(z-\tilde{r}_{n}\right)$. Moreover $g_{\Delta_{n}}(z ; \infty)$ and $g_{\Delta_{n}}(z ; 0)$ are the Green functions of $\Delta_{n}$ with pole at infinity and at zero respectively. Note $g_{\Delta_{n}}(z ; \infty)=$ $\log \left|\Phi_{\Delta_{n}}(z)\right|$.

For a given Borel measure $\mu$ we let $V_{\mu}(z)=\int \log |z-t|^{-1} \mathrm{~d} \mu(t)$ be its logarithmic potential.

With this notation, we can formulate:

Theorem 4.2. Let $d\left(z, \Delta_{n}\right)$ denote the distance from $z$ to $\Delta_{n}$. Then

$$
\pi d\left(z, \Delta_{n}\right) K_{n}\left(z, \omega_{\gamma}\right) \leq\left|\Phi_{\Delta_{n}}(z)\right| \exp \left\{\omega_{n}-2 V_{\mu_{n}}(z)\right\}
$$


where $\mu_{n}$ is the solution of the following equilibrium potential problem

$$
2 V_{\mu_{n}}(x)+x^{\gamma}+x^{-\gamma}+\lambda_{n} \log x \begin{cases}=\omega_{n} ; & x \in \operatorname{supp}\left(\mu_{n}\right) \\ \geq \omega_{n} ; & x \in \mathbb{R}^{+} \backslash \operatorname{supp}\left(\mu_{n}\right)\end{cases}
$$

with $\omega_{n}$ the corresponding equilibrium constant.

Furthermore, it holds $\forall z \in \mathbb{C}$ that

$$
\theta_{n}\left(z ; f_{\gamma}\right)+2 V_{\mu_{n}}(z)+\left[2 n-\lambda_{n}\right] g_{\Delta_{n}}(z ; \infty)+\lambda_{n}\left[\log |z|+g_{\Delta_{n}}(z ; 0)\right]=\omega_{n},
$$

where $\theta_{n}\left(z ; f_{\gamma}\right)$ is the solution of the Dirichlet problem for the function $f_{\gamma}(x)=$ $x^{\gamma}+x^{-\gamma}$ on $\Delta_{n}$ which is given by

$$
\theta_{n}\left(z ; f_{\gamma}\right)=\frac{\operatorname{Re} \sqrt{T_{n}(z)}}{\pi} \int_{\tilde{r}_{n}}^{\tilde{R}_{n}} \operatorname{Re}\left\{\frac{1}{z-t}\right\} \frac{f_{\gamma}(t)}{\sqrt{\left(\tilde{R}_{n}-t\right)\left(t-\tilde{r}_{n}\right)}} \mathrm{d} t .
$$

These two theorems enable us to state the following

THEOREM 4.3. Let $\{p(n)\}_{n \in \mathbb{N}}$ be a sequence of nonnegative integers with $0 \leq$ $p(n) \leq 2 n-1$. Assume that (2.9) holds. Let $I_{n}(f)$ be the $n$-point Gauss-type quadrature formula in $\Lambda_{-p(n), q(n)}$ with $q(n)=2 n-1-p(n)$ for the distribution $\mathrm{d} \alpha(x)=\omega_{\gamma}(x) \mathrm{d} x, x \in[0, \infty)$ with $\omega_{\gamma}(x)$ given by (4.1). Then, for any function $f$ satisfying (2.4) it holds that

$$
\varlimsup_{n \rightarrow \infty}\left|I_{\alpha}(f)-I_{n}(f)\right|^{1 / 2 n} \leq \max \left\{\left(\sigma_{R}\left(f^{+} ; \frac{1}{2(1-\theta)}\right)\right)^{1-\theta},\left(\sigma_{r}\left(f^{-} ; \frac{1}{2 \theta}\right)\right)^{\theta}\right\}
$$

Proof. By Part i) of Theorem 4.1, the functions $R_{x}$ and $r_{x}$ as defined in Section 2 can here be taken to be $(K>1)$

$$
R_{x}=\left(K^{\gamma} \frac{2(1-\theta)}{\gamma B(\gamma)} x\right)^{1 / \gamma} \text { and } \frac{1}{r_{x}}=\left(K^{\gamma} \frac{2 \theta}{\gamma B(\gamma)} x\right)^{1 / \gamma}
$$

Thus

$$
\psi(\theta)=\varlimsup_{n \rightarrow \infty} \frac{R_{v(n)}}{R_{\frac{n}{2(1-\theta)}}}=\varlimsup_{n \rightarrow \infty}\left(\frac{v(n)}{n} 2(1-\theta)\right)^{1 / \gamma}=1
$$

by (2.10). On the other hand

$$
\psi^{*}(\theta)=\varliminf_{n \rightarrow \infty} \frac{r_{v^{*}(n)}}{r_{\frac{n}{2(1-\theta)}}}=\varliminf_{n \rightarrow \infty}\left(\frac{n}{v^{*}(n) 2 \theta}\right)^{1 / \gamma}=1 .
$$

The proof now follows by Theorem 3.5.

THEOREM 4.4. Let $f$ be a function as given by (2.4) with nonnegative coefficients. Assume that $I_{\alpha}(f)<\infty$. Then, under the same conditions as in Theorem 4.3, it holds that

$$
\varlimsup_{n \rightarrow \infty}\left|I_{\alpha}(f)-I_{n}(f)\right|^{1 / 2 n} \geq \max \left\{\eta^{+}, \eta^{-}\right\}
$$

with

$$
\eta^{+}=\left[\frac{\sigma_{R}\left(f^{+} ; \frac{1}{2(1-\theta)}\right)}{4 K \mathrm{e}^{1 / \gamma}}\right]^{1-\theta} \quad \text { and } \quad \eta^{-}=\left[\frac{\sigma_{r}\left(f^{-} ; \frac{1}{2 \theta}\right)}{4 K \mathrm{e}^{1 / \gamma}}\right]^{\theta}
$$

with $K$ given by (4.4). 
Proof. By Corollary 3.9, we only need lower bounds for $\mu(\theta)$ and for $\kappa(\theta)$ which were defined in (2.14) and (2.17) respectively. We have

$$
\mu(\theta)=\varlimsup_{n \rightarrow \infty} \frac{\gamma_{n}^{1 / q(n)}}{R_{\frac{q(n)}{2(1-\theta)}}^{2(n)}} \quad \text { with } \quad \gamma_{n}=\int_{0}^{\infty} \hat{h}_{n m}^{2}(x) x^{-p(n)} \omega_{\gamma}(x) \mathrm{d} x .
$$

Thus $\gamma_{n}=\left[\kappa_{n n}\left(\omega_{\gamma}\right)\right]^{-2}$ with $\kappa_{n n}\left(\omega_{\gamma}\right)$ given by (4.2). Now, by (4.4) and Part ii) in Theorem 3.5 , it can be easily deduced that

$$
\mu(\theta)=\frac{1}{4(\mathrm{e} K)^{1 / \gamma}} .
$$

On the other hand, by (2.17) and (4.3), one has

$$
\kappa(\theta)=\varlimsup_{n \rightarrow \infty} \frac{r_{\frac{p(n)}{2 \theta}}}{\left[K_{n}\left(0, \omega_{\gamma}\right)\right]^{1 / p(n)}}=\varlimsup_{n \rightarrow \infty} \frac{r_{\frac{p(n)}{2 \theta}}}{\left[\tilde{K}_{n}\left(0, \omega_{\gamma}\right)\right]^{1 / p(n)}} .
$$

Here $K_{n}\left(z, \omega_{\gamma}\right)$ denotes the Christoffel function associated with $x^{-(p(n)+1)} \omega_{\gamma}(x)$, while $\tilde{K}_{n}\left(z, \omega_{\gamma}\right)$ represents the one corresponding to $x^{-p(n)} \omega_{\gamma}(x)$. For our purposes, we can use $\tilde{K}_{n}\left(z, \omega_{\gamma}\right)$ instead of $K_{n}\left(z, \omega_{\gamma}\right)$.

Now recall that $\Delta_{n}=\left[\tilde{r}_{n}, \tilde{R}_{n}\right]$, hence $d\left(0, \Delta_{n}\right)=\tilde{r}_{n}$. Thus, by Theorem 4.2 , it follows

$$
\tilde{K}_{n}\left(0, \omega_{\gamma}\right) \leq \frac{\left|\Phi_{\Delta_{n}}(0)\right|^{2 n+1-p(n)}}{\pi \tilde{r}_{n}} \mathrm{e}^{p(n) \lim _{z \rightarrow 0}\left(\log |z|+g_{\Delta_{n}}(z ; 0)\right)} \mathrm{e}^{\theta_{n}\left(0, f_{\gamma}\right)}
$$

Therefore

$$
\frac{r_{\frac{p(n)}{2 \theta}}}{\tilde{K}_{n}\left(0, \omega_{\gamma}\right)^{1 / p(n)}} \geq \frac{r_{\frac{p(n)}{2 \theta}} \pi^{1 / p(n)} \tilde{r}_{n}^{1 / p(n)} \exp \left\{-\frac{\theta_{n}\left(0, f_{\gamma}\right)}{p(n)}\right\}\left|\Phi_{\Delta_{n}}(0)\right|}{\left|\Phi_{\Delta_{n}}(0)\right|^{\frac{2 n+1}{p(n)}} \exp \left\{\lim _{z \rightarrow 0}\left(\log |z|+g_{\Delta_{n}}(z, 0)\right)\right\}}
$$

It is known that

$$
\lim _{|z| \rightarrow \infty}\left\{g_{[a, b]}(z ; \infty)-\log |z|\right\}=-\log \operatorname{Cap}[a, b]=-\log \frac{b-a}{4},
$$

( $\log$ Cap is the logarithmic capacity) and since $g_{\Delta_{n}}(z ; 0)=g_{\Delta_{n}^{-1}}(1 / z ; \infty)$, (we denote $\left.\Delta_{n}^{-1}=\left\{z: 1 / z \in \Delta_{n}\right\}\right)$

$$
\lim _{z \rightarrow 0}\left\{g_{\Delta_{n}^{-1}}(1 / z ; \infty)+\log |z|\right\}=-\log \operatorname{Cap} \Delta_{n}^{-1}
$$

which gives

$$
\lim _{z \rightarrow 0}\left\{g_{\Delta_{n}}(z ; 0)+\log |z|\right\}=-\log \frac{\tilde{R}_{n}-\tilde{r}_{n}}{4 \tilde{R}_{n} \tilde{r}_{n}} .
$$

By the definition of $\theta_{n}\left(z ; f_{\gamma}\right)$, we have

$$
\theta_{n}\left(0 ; f_{\gamma}\right)=-\sqrt{\tilde{R}_{n} \tilde{r}_{n}} \tilde{R}_{n}^{\gamma}\left\{\frac{B_{n}(\gamma-1)}{\tilde{R}_{n}}+\frac{B_{n}(\gamma)}{\left(\tilde{R}_{n} \tilde{r}_{n}\right)^{\gamma+\frac{1}{2}}}\right\}
$$

where

$$
B_{n}(\gamma)=\frac{1}{\pi} \int_{\frac{\tilde{r}_{n}}{R_{n}}}^{1} \frac{t^{\gamma}}{\sqrt{1-t} \sqrt{t-\tilde{r}_{n} / \tilde{R}_{n}}} \mathrm{~d} t
$$

and it holds that

$$
B(\gamma):=\lim _{n \rightarrow \infty} B_{n}(\gamma)=\frac{1}{\sqrt{\pi}} \frac{\Gamma(\gamma+1 / 2)}{\Gamma(\gamma+1)}=\frac{1}{\pi} \int_{0}^{1} \frac{x^{\gamma}}{\sqrt{x(1-x)}} \mathrm{d} x .
$$


Therefore

$$
\lim _{n \rightarrow \infty} \frac{\theta_{n}\left(0 ; f_{\gamma}\right)}{\tilde{R}_{n}^{\gamma}}=\frac{-B(\gamma)}{\lim _{n \rightarrow \infty}\left(\tilde{R}_{n} \tilde{r}_{n}\right)^{\gamma}}=-B(\gamma) \frac{\theta}{1-\theta}
$$

or equivalently

$$
\lim _{n \rightarrow \infty} \theta_{n}\left(0, f_{\gamma}\right) \tilde{r}_{n}^{\gamma}=-B(\gamma)
$$

Thus, by (4.5) and (4.7), we find after some calculations that

$$
\varlimsup_{n \rightarrow \infty} \frac{\tilde{r}_{\frac{p(n)}{2 \theta}}}{\tilde{K}_{n}\left(0, \omega_{\gamma}\right)^{1 / p(n)}} \geq \frac{1}{4 K \mathrm{e}^{1 / \gamma}} .
$$

This proves the theorem.

To end this section, we apply the results for the family of distributions (4.1) to certain integrands $f$. We consider integrands which can be described as follows. Let

$$
f_{1}(z)=\sum_{k=0}^{\infty} c_{k} z^{k} \quad \text { and } \quad f_{2}(z)=\sum_{k=0}^{\infty} b_{k} z^{-k}
$$

be given, with $f_{1}(z)$ and $f_{2}(1 / z)$ entire functions. Then consider integrands $f(z)=$ $f_{1}(z) f_{2}(z)$, so that

$$
f(z)=f^{+}(z)+f^{-}(z)=\sum_{j=0}^{\infty} f_{j}^{+} z^{j}+\sum_{j=1}^{\infty} f_{j}^{-} z^{-j}
$$

where

$$
f_{j}^{+}=\sum_{k=0}^{\infty} c_{k+j} b_{k}, j \geq 0, \quad \text { and } \quad f_{j}^{-}=\sum_{k=0}^{\infty} c_{k} b_{k+j}, j \geq 1 .
$$

In order to fix the ideas, let us take

$$
\begin{aligned}
& f_{1}(z)=\mathrm{e}^{\alpha z}=\sum_{k=0}^{\infty} \frac{\alpha^{k}}{k !} z^{k}, \quad \alpha \in \mathbb{R} \\
& f_{2}(z)=\cos (\beta / z)=\sum_{k=0}^{\infty} \frac{\beta^{2 k}}{(2 k) !}(-1)^{k} z^{-2 k}, \quad \beta \in \mathbb{R} .
\end{aligned}
$$

Thus

$$
\begin{gathered}
f_{2 k}^{-}=(-1)^{k} \beta^{2 k} \sum_{l=0}^{\infty} \frac{(\alpha \beta)^{2 l}}{(2 l) !(2(k+l)) !}(-1)^{l}, \\
f_{2 k+1}^{-}=(-1)^{k+1} \beta^{2 k+1} \sum_{l=0}^{\infty} \frac{(\alpha \beta)^{2 l+1}}{(2 l+1) !(2(k+l+1)) !}(-1)^{l},
\end{gathered}
$$

and

$$
f_{k}^{+}=\alpha^{k} \sum_{l=1}^{\infty} \frac{(\alpha \beta)^{l}}{(2 l) !(2 l+k)) !}(-1)^{l}
$$

Now by (4.4), we can write

$$
R_{x}=\left(K^{\prime} x\right)^{1 / \gamma}, \quad \text { with } \quad K^{\prime}=K^{\gamma} \frac{2(1-\theta)}{\gamma B(\gamma)}, \quad K>1
$$


and

$$
\frac{1}{r_{x}}=\left(K^{\prime \prime} x\right)^{1 / \gamma}, \quad \text { with } \quad K^{\prime \prime}=K^{\gamma} \frac{2 \theta}{\gamma B(\gamma)}, \quad K>1,
$$

where $B(\gamma)$ is given in Theorem 4.1. We can deduce $(s>0)$

$$
\sigma_{R}\left(f^{+}, s\right)= \begin{cases}\infty, & \gamma<1 \\ |\alpha| K^{\prime} s, & \gamma=1 \\ 0, & \gamma>1\end{cases}
$$

while

$$
\sigma_{r}\left(f^{-}, s\right)= \begin{cases}\infty, & \gamma<1 \\ |\beta| K^{\prime \prime} s, & \gamma=1 \\ 0, & \gamma>1\end{cases}
$$

So, by Theorem 4.3, we have for any $\alpha, \beta \in \mathbb{R}$ and $\gamma>1$,

$$
\lim _{n \rightarrow \infty}\left|I_{\alpha}(f)-I_{n}(f)\right|^{1 / 2 n}=0 .
$$

If $\gamma=1$, then one has:

$$
\varlimsup_{n \rightarrow \infty}\left|I_{\alpha}(f)-I_{n}(f)\right|^{1 / 2 n} \leq \max \left\{\left(\frac{|\alpha| K^{\prime}}{2(1-\theta)}\right)^{1-\theta},\left(\frac{|\beta| K^{\prime \prime}}{2 \theta}\right)^{\theta}\right\} .
$$

When considering the so-called balanced situation where $p(n)=n$ for all $n$ (see [JNT83]), then obviously $\theta=1 / 2$ and we then have

$$
\lim _{n \rightarrow \infty}\left|I_{\alpha}(f)-I_{n}(f)\right|^{1 / 2 n} \leq \max \left\{\sqrt{|\alpha| K^{\prime}}, \sqrt{|\beta| K^{\prime \prime}}\right\}=\sqrt{K} \max \{\sqrt{|\alpha|}, \sqrt{|\beta|}\}
$$

where the last equality holds because of the relation between $K, K^{\prime}$ and $K^{\prime \prime}$.

\section{References}

[BDMGVO97a] A. Bultheel, C. Díaz-Mendoza, P. González-Vera, and R. Orive, Quadrature on the half line and two-point Padé approximants to Stieltjes functions. Part II: Convergence, J. Comput. Appl. Math. 77 (1997), 53-76.

[BDMGVO97b] A. Bultheel, C. Díaz-Mendoza, P. González-Vera, and R. Orive, Quadrature on the half line and two-point Padé approximants to Stieltjes functions. Part III: The unbounded case, J. Comput. Appl. Math. 87 (1997), 95-117.

[BDMGVO98] A. Bultheel, C. Díaz-Mendoza, P. González-Vera, and R. Orive, On the convergence of certain Gauss-type quadrature formulas for unbounded intervals, Math. Comp. (1998), Accepted.

[BHMF96] M. Bello-Hernández and A. Martínez-Finkelshtein, Zero asymptotics of Laurent orthogonal polynomials, J. Approx. Theory 85 (1996), 324-342.

[JNT83] W.B. Jones, O. Njăstad, and W.J. Thron, Two-point Padé expansions for a family of analytic functions, J. Comput. Appl. Math. 9 (1983), 105-124.

[LLMF95] G. López-Lagomasino and A. Martínez-Finkelshtein, Rate of convergence of twopoint Padé approximants and logarithmic asymptotics of Laurent-type orthogonal polynomials, Constr. Approx. 11 (1995), 255-286.

[Lub83] D. Lubinsky, Geometric convergence of Lagrangian interpolation and numerical integration rules over unbounded contours and intervals, J. Approx. Theory 39 (1983), 338-360.

[Mha96] H.N. Mhaskar, Weighted polynomial approximation, World Scientific, 1996.

[Rud74] W. Rudin, Real and complex analysis, 2nd ed., McGraw-Hill, New York, 1974. 
Department of Computer Science, K.U.Leuven. Leuven, Belgium

E-mail address: Adhemar.Bultheel@cs.kuleuven. ac.be

$U R L:$ http://www.cs.kuleuven.ac.be/ ${ }^{\sim}$ ade/

Department Análisis Math., Univ. La Laguna, Tenerife, Spain

E-mail address: cjdiaz@ull.es

Department Análisis Math., Univ. La Laguna, Tenerife, Spain E-mail address: pglez@ull.es

Department Análisis Math., Univ. La Laguna, Tenerife, Spain

E-mail address: rorive@ull.es 